\section{Evidence for Activated Carrier Mobility in Organic Solids}

STEADY state space charge limited currents (SCLC) were obtained at different temperatures for a series of dipyridylium model compounds (Fig. 1) into which one TCNQ molecule (tetracyanoquinodimethane), in the form of a radical ion, was introduced. Some compounds were produced containing two molecules of TCNQ, one associated with each ring. Polymers of these compounds were also produced and SCLC measured (details of preparation will be published elsewhere).
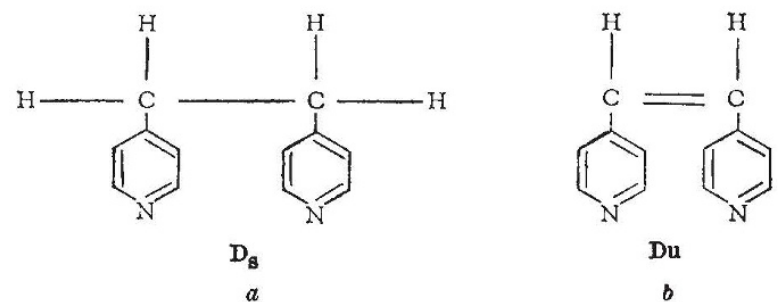

Fig. 1. Chemical structure of $(a)$ 1,2-bis-(4-pyridyl)-ethane, $\mathrm{D}_{\mathrm{8}}$, and (b) 1,2-bis-(4-pyridyl)-ethylene, Du.

The conductivity measurements were made on 0.5 inch diameter cylindrical pellets. Pellets prepared under pressures in vacuo between 20,000 and 100,000 pounds/ inch had essentially identical conductivities. Electrical contact was made with vacuum-deposited gold electrodes or, in some cases, contacts were applied by covering the top and bottom surfaces with a thin layer of gold powder followed by recompression in the hydraulic press. Both processes resulted in firmly adherent, cohesive, contacts which were ohmic at voltages below those at which appreciable charge injection occurred. In one case only was it possible to carry out measurements on a single crystal; the resulting activation energy, $0.129 \mathrm{eV}$, was close to that obtained with the compactions, namely, $0 \cdot 103 \mathrm{eV}$.

The concentrations of free carriers at thermal equilibrium, $n_{c o}$, were obtained ${ }^{1}$ from the transition voltage $V_{t r}$ from ohmic to parabolic voltage dependence, evaluated graphically using the following equation

$$
n_{c o}=\frac{2 \varepsilon \varepsilon_{0} \mathrm{~V}_{t r}}{e t^{2}}
$$

where $e$ is the charge of the electron, $\varepsilon_{0}$ the permittivity of free space, $\varepsilon$ the relative permittivity and $t$ is the interelectrode spacing. With knowledge of the carrier concentration and the conductivity $\sigma$, the mobility may then be obtained from

$$
\mu=\frac{\sigma}{e n_{c o}} \mathrm{~cm}^{2} \mathrm{~V}^{-1} \mathrm{~s}^{-1}
$$

The results are summarized in Fig. 2. It is seen that the carrier concentration remains substantially constant, to within an order of magnitude, at about $10^{11} \mathrm{~cm}^{-3}$, over a temperature range in which the resistivities, some of which are also shown, change by up to eight orders of magnitude. In view of the uncertainties involved in the graphical location of $\mathrm{V}_{t r}$ and the probable changes in the effective permittivity at low temperatures, the values of $n_{c o}$ are estimated to be accurate only to within a factor of five. The largest apparent change in $n_{c o}$ was found in the unsaturated Du 1-TCNQ compound, which appenred to drop from $4.5 \times 10^{11}$ at $-187^{\circ} \mathrm{C}$ to $1.2 \times 10^{10}$ at $-78^{\circ} \mathrm{C}$. In that temperature interval, the conductivity increased by five orders of magnitude.

The temperature dependence of the mobility deduced from SCLC data could be fitted to a shallow trap model ${ }^{2}$, but this would require mobilities $\geq 40,000 \mathrm{~cm}^{2} / V$ s. Such values would be substantially larger than those shown in Fig. 2 or found from Hall mobility measurements (to be published elsewhere) and larger than those

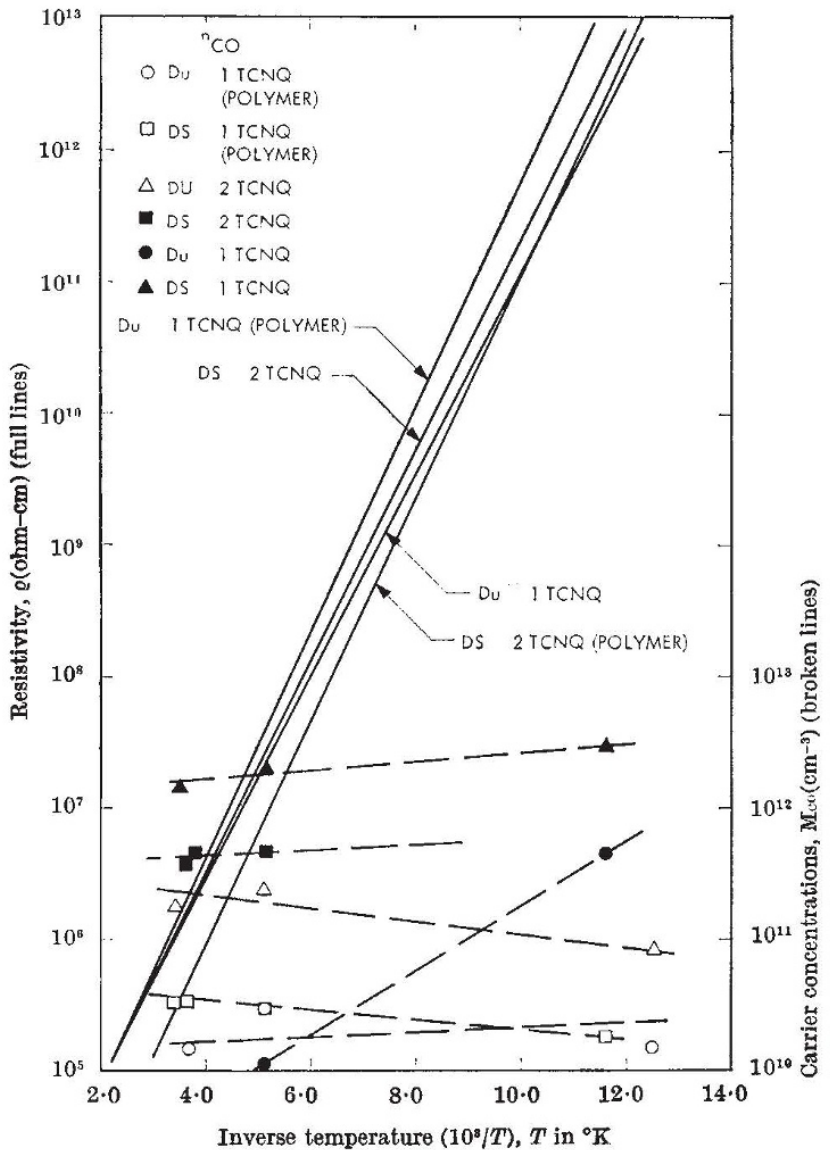

Fig. 2. Temperature dependence of resistivity, $\varrho$, and concentration of carriers, $n_{00}$

found in most single crystal inorganic semiconductors. Mobilities as large as this are extremely unlikely in systems lacking long range order.

We are forced to the conclusion that the observed conductivity changes are caused by mobility changes. Such a thermally activated mobility in organic materials has been repeatedly proposed ${ }^{3}$, but to our knowledge this work presents the first evidence for its existence.

It is of interest that the carrier concentration values appear to be invariant-again to plus or minus an order of magnitude-with respect to saturation versus unsaturation, introduction of a second TCNQ molecule and polymerization. In fact, carrier concentrations of the order reported here have also been observed by other workers ${ }^{4}$.

This article presents the results of one phase of research carried out at the Jet Propulsion Laboratory, California Institute of Technology, sponsored by the US National Aeronauties and Space Administration.
F. Gutmann
A. M. HermanN
A. Rembaum

Jet Propulsion Laboratory,

California Institute of Technology,

Pasadena, California.

Received November 29, 1968; revised January 28, 1969.

${ }^{1}$ Lampert, M. A., Rose, A., and Smith, R. W., J. Phys. Chem. Solids, 8, 464 (1959)

${ }^{2}$ Rose, A., Phys. Rev., 97, 1538 (1955).

${ }^{3}$ Frolich, H., and Sewell, G. L., Proc. Phys. Soc., 74, 643 (1959); Tredgold, R. H., ibid., 80, 807 (1962). Pohl, H. A., and Opp, D. A., J. Thys. Chem., 66, 2121 (1962). Hadek, V., and Ulbert, K., Coll. Czech. Chem. Commun., 32, 1118 (1967). Hermann, A. M., and Rembaum, A., $J$ Polymer Sci., Part C, Polymer Symposia, 17, 120 (1967). Cherry, R. J.,
Quart. Rev.,22, 162 (1968).

${ }^{4}$ Gutmann, F., and Lyons, L. E., Organic Semiconductors (John Wiley and Sons, New York, 1967). 